EPJ Web of Conferences 103, 10003 (2015)

DOI: $10.1051 /$ epjconf/ 201510310003

(C) Owned by the authors, published by EDP Sciences, 2015

\title{
Response of Graphene Based Gated Nanodevices Exposed to THz Radiation
}

\author{
G.E. Fedorov ${ }^{1 a}$, I.A. Gaiduchenko ${ }^{1}$, A.D. Golikov ${ }^{1}$, M.G. Rybin² ${ }^{2}$, E.D. Obraztsova ${ }^{2}$, B.M. Voronov ${ }^{1}$, D. \\ Coquillat $^{3}$, N. Diakonova ${ }^{3}$, W. Knap ${ }^{3}$, and G.N. Goltsman ${ }^{1,4}$ \\ ${ }^{1}$ Moscow State Pedagogical University, 1 Malaya Pirogovskaya, 119991, Moscow, \\ ${ }^{2}$ Prokhorov General Physics Institute of the Russian Academy of Sciences 38 Vavilov Str., 119991, Moscow, \\ 3 Laboratoire Charles Coulomb UMR 5221, Université Montpellier 2, F-34095, Montpellier, France \\ ${ }^{4}$ National Research University Higher School of Economy, 20 Myasnitskaya, 101000, Moscow
}

\begin{abstract}
In this work we report on the response of asymmetric graphene based devices to subterahertz and terahertz radiation. Our devices are made in a configuration of a field-effect transistor with conduction channel between the source and drain electrodes formed with a CVD-grown graphene. The radiation is coupled through a spiral antenna to source and top gate electrodes. Room temperature responsivity of our devices is close to the values that are attractive for commercial applications. Further optimization of the device configuration may result in appearance of novel terahertz radiation detectors.
\end{abstract}

Keywords: graphene, terahertz radiation, terahertz radiation detection.

Terahertz radiation has uses in applications ranging from security to medicine. However, sensitive room-temperature detection of terahertz radiation is notoriously difficult. The use of nano-scale objects is very attractive to invent and discover cost-effective solutions for new THz detectors. One particular route is the use of carbon nanomaterials: single-wall carbon nanotubes (SWCNTs) and graphene and it already brought very encouraging results even at room temperature [1-3]. Here we report on the DC voltage response of asymmetric graphene based devices to sub-terahertz and terahertz radiation in the frequency range of $130 \mathrm{GHz}$ to $0.5 \mathrm{THz}$.
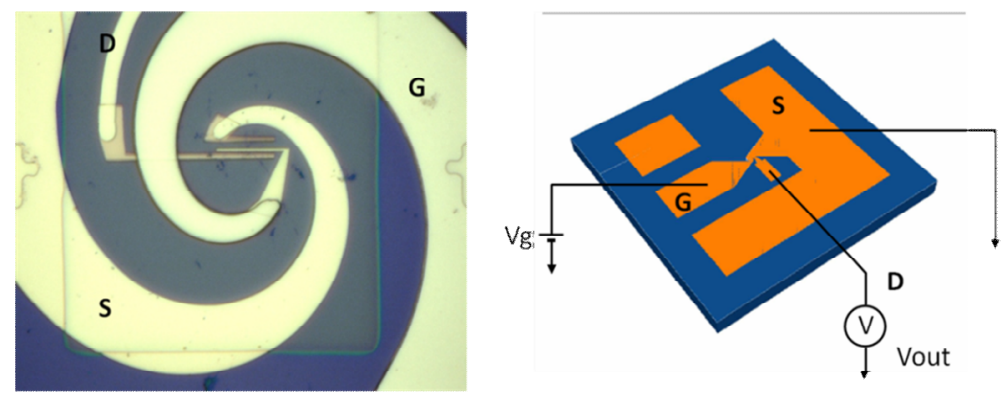

Figure 1. Optical image (left) and the electrical scheme of a graphene based THz detector.

${ }^{a}$ Corresponding author: gefedorov@mail.ru

This is an Open Access article distributed under the terms of the Creative Commons Attribution License 4.0, which permits unrestricted use, distribution, and reproduction in any medium, provided the original work is properly cited. 
Our devices are made in a configuration of a field-effect transistor. The conduction channel between the source and drain electrodes is formed with a CVD-grown graphene. The radiation is coupled to the device through a spiral antenna to source and top gate electrodes. Details of the sample fabrication procedure will be described in another paper. Importantly the technological route implied in our work is compatible with traditional microelectronic procedures and is therefore scalable for further mass production.

The typical transistor characteristic of our devices is shown in Figure 2a. The conductance minimum is associated with alignment of the Fermi level with the so called Dirac point of graphene bandstructure. As any of the devices is exposed to radiation with a power $P$ of $400 \mu \mathrm{W}$ at a frequency of $140 \mathrm{GHz}$ the IV curve shifts, so that the absolute value of the current at zero bias voltage equals about $0.1 \mu \mathrm{A}$. The zero-current crossing occurs at a bias voltage $V_{0}$ of about $0.5 \mathrm{mV}$. This clearly indicates the appearance of a DC voltage induced by the radiation equal to $V_{0}$, which we further refer to as the response voltage $V_{R E S P}$. As it has been discussed in [4] the response of such a device occurs due to excitation of plasma waves (PW). When the propagation length of these waves is much shorter than the channel length the responsivity $R=V_{R E S P} / P$ of the device will be proportional to its transconductance $d G / d V_{G}$ and inversely proportional to its conductance, $G$ :

$$
R \sim \frac{d G}{d V_{G}} \times \frac{1}{G}
$$

As seen from the figure $2 b$ the dependence of the response voltage is well described by the above relation. The maximum magnitude of the responsivity is about $1 \mathrm{~V} / \mathrm{W}$ which is larger than in case of similar devices as reported in [4]. This is possibly due to better coupling of the device to the radiation achieved by feeding the radiation through a silicon lens.

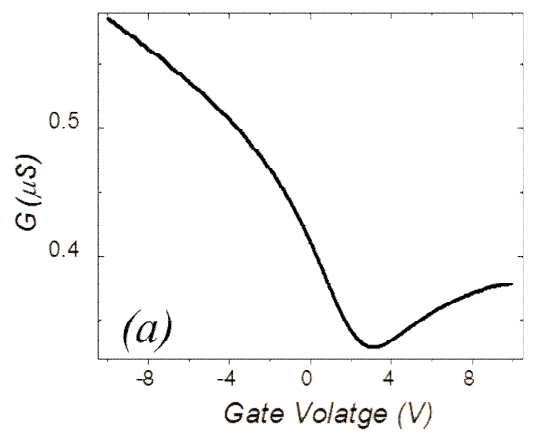

Figure 2. (a) Transistor characteristic of a typical device. the device transconductance and resistance.

Thus we maintain good match between the measured dependence of the response voltage on the gate voltage and the one calculated based on the transistor characteristic. This in turn means applicability of the PW scenario. It implies that the responsivity of the device will increase if the ratio PW propagation length over the channel length. Further optimization of the device may be achieved through decreasing the channel length or through increasing its mobility.

This work was supported by the Grant of Ministry of Education and Science of Russian Federation under Contract No. 14.586.21.0003 (project ID RFMEFI58614X0003).

\section{References}

1. G. Fedorov et al., Appl. Phys. Lett. 103, 181121 (2013)

2. J. Kono et al, Nano Letters 4 (7) pp 3953-58 (2014)

3. M. S. Fuhrer et al, Nature Nanotechnology, 9 pp 814-19 (2014)

4. L.Vicarelli, et al. Nature Mater. 11, 865-871 (2012) 